REVIEW ARTICLE

\author{
B.A. Georgy
}

\section{Metastatic Spinal Lesions: State-of-the-Art Treatment Options and Future Trends}

\begin{abstract}
SUMMARY: The purpose of this article is to review the current state of the art for treating symptomatic spinal fractures associated with malignant lesions and to present potential future trends in treatments for this patient population. Epidemiology, clinical presentation, and biomechanical ramifications of these lesions are summarized and treatment regimes, clinical outcomes, and complications and technical issues associated with treatments are presented. Potential future trends and new technologies for performing vertebral body augmentation in patients with metastatic spinal lesions are also discussed.
\end{abstract}

M etastatic bone tumors in the spine are painful and debilitating but are challenging to treat and often require relatively extensive surgery, which many clinicians and patients are reluctant to pursue. Percutaneous approaches for performing vertebral body augmentation for treating metastatic spinal lesions have been developed as good alternatives to open surgery. These types of procedures have evolved immensely over the last 10 years, and the current state of the art has been found to fit neatly into the conventional oncologic treatment algorithm.

\section{Epidemiology and Clinical Presentation}

Spinal metastasis is the most commonly encountered tumor of the spine, ${ }^{1}$ occurring in $\leq 40 \%$ of patients with cancer. ${ }^{2}$ Each year, $5 \%$ of patients with cancer, or approximately 61,000 persons, will develop spinal metastasis. ${ }^{3}$ The cancers most often metastasizing to the spine include breast (21\%), lung (14\%), prostate $(8 \%)$, renal (5\%), gastrointestinal (5\%), and thyroid (3\%). Two of 3 patients are affected in the thoracic spine, whereas affected areas of the other third of patients are split 1:2 in the cervical and lumbar regions, respectively. ${ }^{2}$ The posterior half of the vertebral body is usually infiltrated first, with the anterior body, lamina, and pedicles becoming involved later. ${ }^{4}$ The treatment regimen for spinal metastasis is generally palliative and consists of a combination of medical therapy (steroids, pain medication, and chemotherapy), radiation therapy, and surgery.

The primary goals of treatment are to relieve pain and preserve or restore function when managing patients affected by spinal metastasis. Patients with metastatic spine disease typically present with progressive and unrelenting pain that is not alleviated with rest and is often worse at night. Pain is generally described in at least 1 of 3 ways: 1) constant and localized, 2) radicular, or 3) axial, coinciding with functional disability. Localized pain is generally thought to be a result of periosteal stretch occurring with tumor expansion and is usually treated by using radiation because this therapy is effective in decreasing the tumor size. Radicular pain, most likely due to the tumor pressing against the nerve root, is also addressed by using radiation therapy but can be treated by using nerve root blocks as well. Axial pain, most frequently associated with mechanical

Received January 16, 2008; accepted after revision February 29.

From Valley Radiology Consultants, Escondido, Calif.

Please address correspondence to Bassem A. Georgy, MD, Interventional Neuroradiology, Assistant Clinical Professor UCSD, Valley Radiology Consultants, 321 West Mission Ave, Escondido, CA 92025; e-mail: bgeorgy@earthlink.net

DOI 10.3174/ajnr.A1137 instability of the spine or pathologic vertebral body fracture, is worsened with physical activity but relieved with rest. Axial pain is generally treated by surgically stabilizing the spine.

\section{Biomechanics of Pathologic Fractures}

In patients with spinal metastasis, pathologic fracture can occur under normal physiologic stress. ${ }^{5}$ Partial or total destruction of the anterior vertebral body results in decreased load-bearing capacity of the spine. How and when pathologic fracture occurs is generally determined by the size and location of the tumor, the extent of tumor destruction, and the patient's bone mineral attenuation. ${ }^{1,6}$ The threshold for pathologic fracture can be accurately predicted by evaluating the relationship between bone mineral attenuation and the cross-sectional area of the intact vertebral body. ${ }^{5,6}$ When the critical threshold is exceeded-corresponding to compromise of $51 \%-96 \%$ of the cross-sectional area, depending on bone mineral attenuation - the vertebral body becomes prone to pathologic fracture.

Krishnaney et $\mathrm{al}^{1}$ postulated that the effects of vertebral body destruction by the tumor are best characterized by graphically representing the affected vertebral body as a series of 27 identically shaped adjacent cubes (Fig 1). Destruction of the middle third of the vertebral body in the axial plane will result in gross instability (Fig 2). In this case, the anterior and middle columns of Denis ${ }^{7}$ are likely to be completely disrupted. In contrast, loss of the middle third in the sagittal plane is not usually associated with significant destabilization. In this case, disruption is limited to one third of the anterior and middle columns of Denis; hence, stability is preserved. Ventrally situated tumors (anterior column) have a potentially greater destabilizing effect than dorsally located masses (middle column) in the presence of intact dorsal elements. ${ }^{7}$

\section{Conventional Treatment Regimen}

\section{Radiation Therapy}

Radiation therapy has a long proved record as a successful means for alleviating pain in patients with metastatic spinal lesions. Analgesic use is decreased, and localized control of the disease is gained with minimal side effects. Tumors considered to be highly radiosensitive include lymphomas, myelomas, seminomas, and neuroblastomas; breast and prostate carcinomas are considered moderately radiosensitive. Earlier studies evaluating the success of radiation therapy showed that neurologic improvement was obtained in $40 \%-50 \%$ of patients, but more recent studies have demonstrated success rates of $70 \% .^{8,9}$ Therapeutic radiation is generally the only option for 

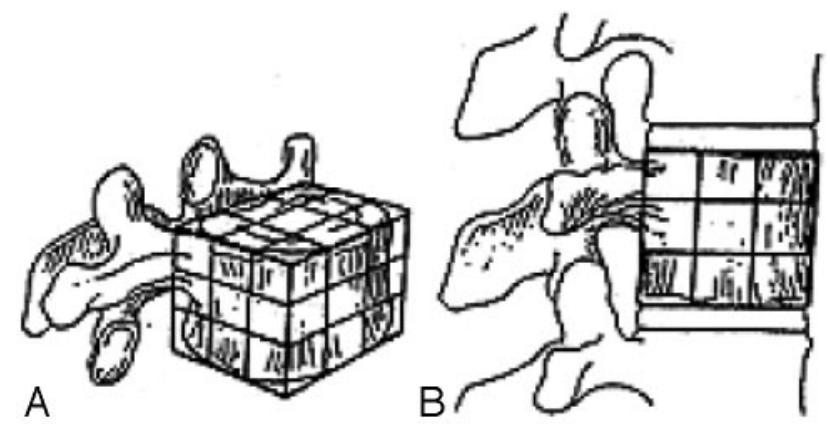

Fig 1. The vertebral body can be depicted as a cube composed of 27 smaller cubes to indicate tumor location: oblique $(A)$ and lateral $(B)$ views. (Reprinted with permission from Elsevier Ltd.)
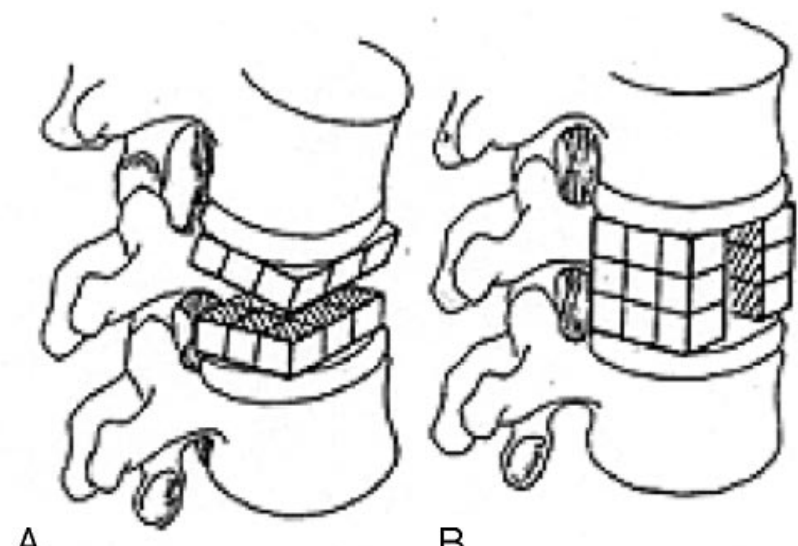

A

B
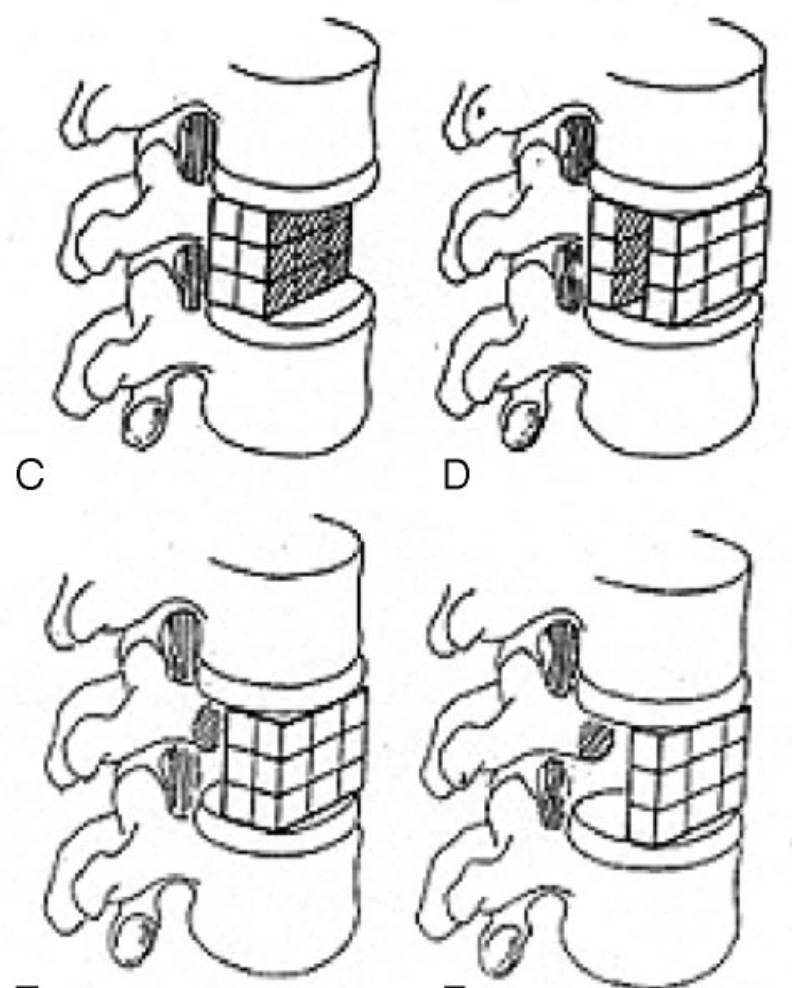

E

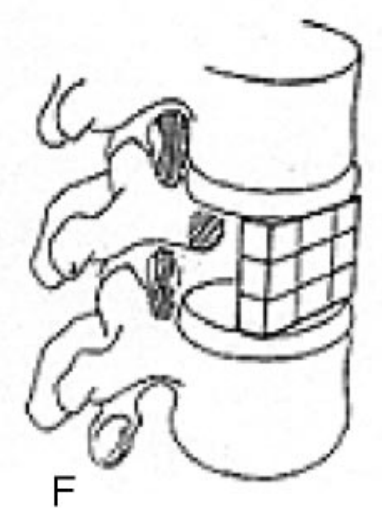

Fig 2. Tumor infiltration or damage to portions of the cube depicted in Fig 1 may destabilize the spine in varying fashions. Destruction of the middle third in the axial plane $(A)$ results in gross instability, whereas destruction of the middle third in the sagittal plane $(B)$ may not be associated with significant destabilization. A lesion in the ventral portion of the vertebral body in the coronal plane $(C)$ affects stability more than a lesion in the middle $(D)$ or dorsal ( $E$ and $F$ ) portions. (Reprinted with permission from Elsevier Ltd.) patients who have radiosensitive tumors, cannot tolerate surgery, or have a poor survival prognosis.

Nevertheless, radiation therapy used as a stand-alone treatment may have some significant shortcomings. Pain relief may be delayed for up to 2 weeks following the procedure, ${ }^{10}$ and this treatment does not correct existing biomechanical abnormalities or stabilize the spine. In addition, it is not effective for preventing imminent vertebral body collapse; almost half of patients undergoing radiation therapy subsequently experience vertebral body compression fractures. ${ }^{11,12}$ Because of these drawbacks, Regine et $\mathrm{al}^{13}$ concluded that when patients meet the clinical criteria for surgery, the role of radiation therapy is that of adjuvant therapy. Adding a spine-stabilization procedure to a radiation therapy program is thought to be critical for managing axial pain and providing neurologic recovery. ${ }^{14}$ Optimally, radiation therapy is best performed following a spine stabilization procedure because the risk of postoperative complications, particularly wound infection and dehiscence, is lessened.

\section{Open Surgery}

Historically, the indications for surgical intervention have included radioresistant disease, spinal instability, spinal cord compression, acute or progressive neurologic deterioration, previous exposure of the spinal cord to radiation, incapacitating pain-despite orthotic treatment or radiation-impending pathologic fracture, and life expectancy of at least 3 months. ${ }^{14}$ The principal objectives of surgery include nerve root decompression, stabilization, and reconstruction of the anatomic spinal column. ${ }^{2}$ Surgery is thought to maximize the patient's quality of life because it is likely to restore or preserve neurologic function as well as relieve pain. Nevertheless, prospective surgical candidates must be considered adequately fit for undergoing a surgical procedure that is associated with a relatively high risk of significant complications.

The surgical approach depends on the location of the tumor, the presence or absence of spinal instability, and the presence or absence of neural compression or neural deficit. In 1989, James Weinstein ${ }^{15}$ proposed a model designed to enhance surgical planning in patients with spinal metastasis. In this model, the vertebral body is delineated into 4 zones (Fig 3 ), which are used to describe the location of the metastatic lesion. Zone I includes the spinous process to the pars interarticularis and superior facet. Zone II encompasses the superior articular facet, transverse process, and the pedicle from the level of the pars to its junction with the vertebral body. Zone III consists of the anterior three quarters of the vertebral body, and zone IV is the posterior one quarter of the vertebral body. Within these zones, the tumor itself is described as intraosseous, extraosseous, or distance metastasis.

Zone I and II lesions are accessed by using a posterior or posterolateral surgical approach. These types of lesions are usually treated by using posterior decompression and stabilization. Zone III lesions are typically accessed by using an anterior surgical approach. This allows direct access to the tumor, and effective reconstruction of the weight-bearing anterior portion of the spinal column can be achieved by using allograft, autograft, cages, plates, or polymethylmethacrylate (PMMA) implants. ${ }^{14}$ Zone IV lesions are the most difficult to treat because they require using a combined anterior and posterior approach. 


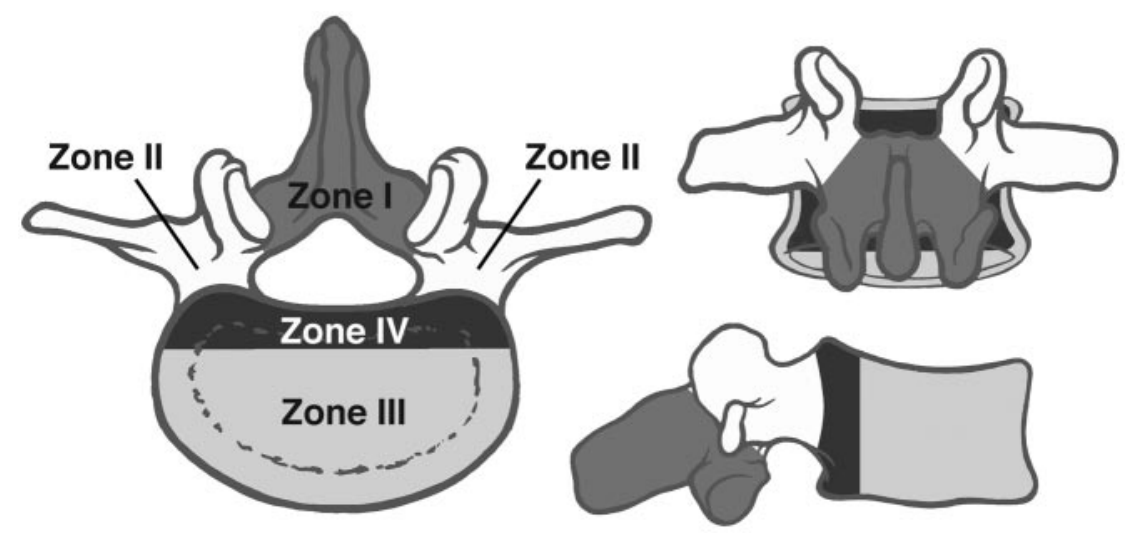

Fig 3. The vertebral body and adjacent structures can be depicted as 4 disparate zones when considering surgery to treat vertebral body tumors.

In rare cases, the metastasis may be isolated to the lamina or the spinous process. In these patients, a posterior approach with decompressive laminectomy, with or without posterior instrumentation, may be indicated. ${ }^{2}$ With this type of procedure, the spinal canal is enlarged with the laminectomy, but the site of spinal cord compression (ie, ventral to the cord) is left unaddressed. If the lesion site is relatively more anterior, spinal instability may not be very easily treated, and the procedure may potentially lead to kyphosis-related pain postoperatively. Subsequently, decompressive laminectomy may not provide better results than using radiation therapy alone, even when combined with adjuvant radiation. ${ }^{9}$ In most of these patients, surgical outcome is improved by using an anterior approach over laminectomy because the risk of perioperative complications and side effects is reduced.

\section{Percutaneous Image-Guided Vertebral Body Augmentation}

Percutaneous vertebral body augmentation, such as vertebroplasty or kyphoplasty, offers a less invasive method than open surgery for treating patients with spinal malignancy. In 1981, Harrington ${ }^{16}$ was the first to propose using bone cement augmentation as a method for relieving pain associated with malignant spinal tumors. In essence, he described a "fusion" type of procedure, in which bone cement was inserted around and into tumor-affected vertebral bodies, after removing portions of the tumor, as a method to stabilize the spine. With this procedure, 13 of 14 patients had excellent pain relief with restoration of spinal stability — clinical benefits persisted through the follow-up period, ranging from 13 to 45 months. Spinal flexion-extension $\mathrm{x}$ rays showed no significant motion between PMMA-augmented vertebrae, suggesting that the procedure could be used successfully to stabilize the spine. Furthermore, future cancer treatments (eg, radiation) were not impacted.

Subsequently, percutaneous vertebral body augmentation procedures such as vertebroplasty and kyphoplasty were developed as a means of managing malignant spinal lesions and were used with relatively good success. During the mid-1990s, Cotten et $\mathrm{al}^{17}$ reported that $97 \%$ of patients had at least some pain relief within the first 48 hours after undergoing a vertebroplasty procedure: $13.5 \%$ were pain free, $55 \%$ showed substantial improvement, and 30\% were moderately improved. Weill et $\mathrm{al}^{18}$ reported that $73 \%$ and $65 \%$, respectively, of 37 patients undergoing vertebroplasty for malignant spinal le- sions had sustained pain relief at 6 and 12 months postoperatively. Deramond et al ${ }^{19}$ suggested moderate-to-complete pain relief in $80 \%$ of 101 patients. Using kyphoplasty for vertebral compression fractures associated with multiple myeloma in 18 patients, Dudeney et $\mathrm{al}^{20}$ reported significantly improved quality of life through at least 52 months as measured by using SF-36 scores. Pflugmacher et $\mathrm{al}^{21}$ reported significantly decreased pain and significantly improved function scores in a series of 20 patients through at least 12 months. Fourney and Gokaslan ${ }^{14}$ reported that $84 \%$ of 56 patients, 21 with myeloma and 35 with primary spinal malignancy, had marked or complete pain relief through at least the first year.

Nevertheless, percutaneous vertebral body augmentation used for treating advanced spinal metastasis has not been widely adopted because it can be technically challenging to perform safely in this population. Most patients with metastatic lesions have epidural extension of the tumor with possible disruption of the posterior cortical border. Complications associated with bone cement extravasation occur more often when treating patients with metastatic disease $(\leq 10 \%)$ than in those with osteoporosis $(1 \%-2 \%)$ or spinal angiomas $(2 \%-$ $3 \%) .{ }^{22}$ Mousavi et $\mathrm{al}^{23}$ reported that the risk of cement leakage during percutaneous vertebroplasty in the metastatic spine is significant, with $\leq 85.7 \%$ of procedures resulting in cement extravasation outside of the vertebral body. However, extravasation in these cases is not usually clinically significant.

The higher risk of extravasation in patients with spinal malignancy, compared with patients with osteoporotic vertebrae, may be attributed to the increased in situ pressures generated during the procedure. ${ }^{24}$ Injection of bone cement into a vertebral body with a resident tumor is more difficult than injection into an osteoporotic vertebral body. The potential for eliciting tumor cell extravasation is a concern and deters many clinicians from using percutaneous vertebral body augmentation as a treatment option for patients with spinal metastasis.

Clearly, percutaneous vertebral body augmentation procedures have been used successfully for alleviating pain and functional disability associated with metastatic spine lesions. This treatment can also be used in conjunction with conventional oncology therapies, including radiation therapy. However, the potentially significant clinical drawbacks have inhibited its use. Subsequently, clinical research in this area has tended to revolve around minimizing the limitations of using conventional verte- 
broplasty or kyphoplasty in this application by developing new techniques for permitting a safe and efficacious procedure.

\section{Adaptations to Conventional Vertebral Body Augmentation}

\section{Radio-Frequency Ablation with Vertebroplasty}

Grönemeyer et $\mathrm{al}^{25}$ were the first to report their clinical experience using radio-frequency ablation of spinal tumors in combination with vertebroplasty. Patients had unresectable osteolytic spine metastases and had not responded to radiation therapy or chemotherapy. The purpose of their study was to determine the feasibility, effectiveness, and safety of using radio-frequency ablation as a means to alleviate pain and back pain-related disability in patients with vertebral and paravertebral spinal tumors. Ten patients were treated by using radiofrequency ablation; 4 patients also underwent vertebroplasty. The procedure was performed under CT and fluoroscopic guidance, in which a multielectrode array (RITA Medical Systems, Mountain View, Calif) was used to thermally ablate $\left(50^{\circ}-120^{\circ} \mathrm{C}\right.$ for $8-12$ minutes) the tumor. No side effects or complications were observed, pain and back pain-related disability were alleviated, and neurologic function was preserved or stabilized. The concept of using the combination of radiofrequency ablation and vertebroplasty caught on quickly as a technically innovative procedure for treating patients with metastatic spinal tumors. Immediately following the report of Grönemeyer et al, ${ }^{25}$ several other investigators reported similarly positive clinical outcomes in individual cases ${ }^{26,27}$ and in small case series ${ }^{26,28}$ using this procedure.

The results for a larger multicenter study $(N=43)$ published in $2004^{29}$ brought to light some of the potential side effects associated with the radio-frequency ablation procedure. This study included 43 patients treated in both Europe and the United States. All patients had pain of $>4$ on a 10-point scale associated with spinal metastasis and had tried and failed, or were ineligible for, conventional treatments such as radiation therapy or surgery for treating spinal metastases. Before radio-frequency ablation treatment, patients had a mean pain score of 7.9 points; by 24 weeks posttreatment, the average pain score was reduced to 1.4 points. Complications observed with the procedure included a second-degree skin burn at the grounding pad site $(n=1)$, transient bowel and bladder incontinence following treatment of a metastasis involving the sacrum $(n=1)$, and a fracture of the acetabulum following radio-frequency ablation of an acetabular lesion $(n=1)$. Longer term follow-up of the same cohort was conducted to evaluate tumor progression and postoperative complications. ${ }^{30}$ The mean time to tumor progression was $730 \pm$ 54 days (Kaplan-Meier estimate); other reported side effects associated with the radio-frequency ablation procedure included increased collateral pain and numbness in the lower limbs, unilateral monoradiculopathy at the same level that radio-frequency ablation had been administered, and paresthesia leading to a fall.

When the posterior wall of the vertebra is compromised by the malignancy, radio-frequency ablation may pose substantial risk for nerve damage because the spinal cord is in relatively close proximity to the ablation field. Buy et a ${ }^{31}$ reported a modified radio-frequency ablation delivery method designed to obviate inferring radio-frequency current into the spinal cord. Three patients with high-risk spinal tumors were treated. A high-risk patient was defined as having a tumor within $1 \mathrm{~cm}$ of a neural structure or involving paravertebral soft tissue. The radio-frequency ablation device consisted of 2 needles (electrodes), which were activated in a bed of hypertonic saline $(5.85 \%)$. This design allows more control over energy delivery than the conventional monopolar radio-frequency ablation technology. Within 24 hours of treatment, all 3 patients reported that pain was alleviated. Results from the postoperative neurologic examination were consistent with those of an uneventful case.

\section{Tumor Debulking Combined with Vertebral Body Augmentation}

In 2006, Tschirhart et al ${ }^{32}$ suggested that tumor-volume reduction (as opposed to tumor necrosis occurring with radio-frequency ablation) be used in concert with vertebroplasty as a useful means for stabilizing vertebral bodies infiltrated by metastatic tumors. Using a biphasic parametric finite element analysis (FEA) model simulating a tumor-infiltrated vertebral body at the lumbar level, they reported that restoration of vertebral stability was theoretically possible after removing $30 \%$ of the tumor and inserting $1-2 \mathrm{~cm}$ of bone cement. The model indicated that creation of a cavity in the vertebra would permit preferential cement deposition in the region affected by lytic disease and would facilitate restoration of stability because the region of lytic destruction and the surrounding bone would both be stabilized.

The following year, Ahn et al ${ }^{33}$ confirmed the FEA model of Tshirchart et $\mathrm{al}^{32}$ in cadaveric tissue. Using laser-induced thermotherapy (LITT) to ablate the tumor immediately before vertebroplasty-facilitated bone cement placement improved biomechanical stability and reduced the risk of cement extravasation over using the vertebroplasty procedure alone. Thoracic spine segments with simulated tumors were treated by using LITT to create a void, which was then filled with bone cement. In this study, the tested spinal motion segments were intact or had simulated metastases; those with simulated metastases had a vertebroplasty procedure, with and without LITT. Spinal motion segments were tested in axial compression to determine posterior wall motion and biomechanical failure. The LITT-treated vertebral bodies showed consistent cement deposition with no evidence of extravasation into the spinal canal. In contrast, vertebral bodies undergoing vertebroplasty alone showed bone cement deposition anteriorly and laterally to the tumor in all cases. Stability of the specimens undergoing LITT with vertebroplasty was greatly improved over that of the vertebroplasty-alone specimens. Using LITT with vertebroplasty reduced posterior wall motion, whereas vertebroplasty alone was associated with increased motion and higher variability between specimens.

Radio-frequency-based plasma ablation has recently become available as a method that can also be used to create a void in vertebral bodies affected by metastasis. This type of technology allows creation of a void in tissue, similar to that using the laser devices, but is recognized to be safer in several clinical applications. With this type of device, radio-frequency energy is used to excite the electrolytes in a conductive medium, such as saline solution, creating precisely focused plasma. The energized particles in the plasma have sufficient energy to break molecular bonds, therefore having the ability to remove soft tissue at relatively low temperatures, typically $40^{\circ}-70^{\circ} \mathrm{C}$. Georgy and Wong ${ }^{34}$ reported the clinical feasibility 

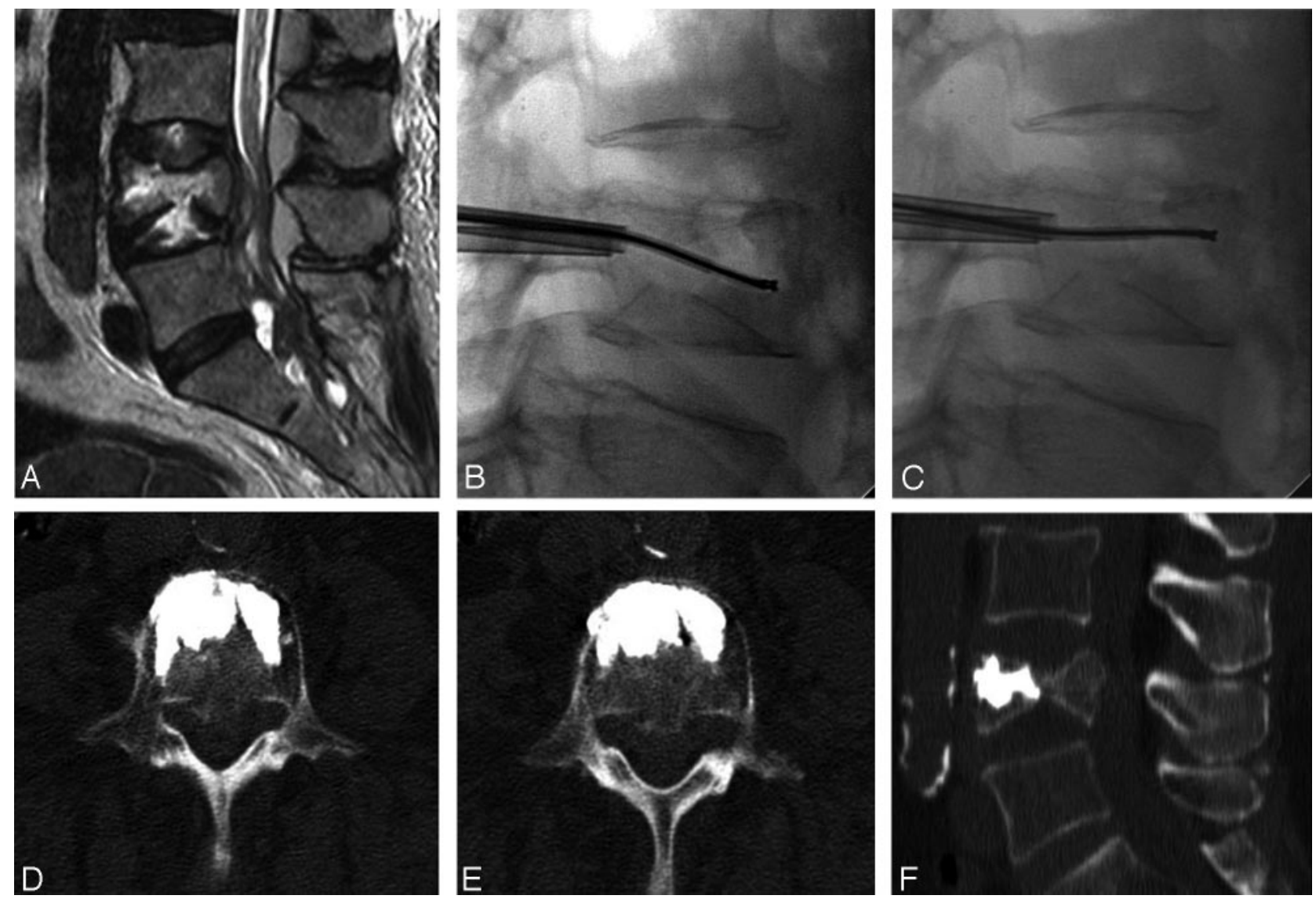

Fig 4. $A, A$ 71-year-old woman with undifferentiated cancer and a lesion at $L 4 . B$ and $C, A$ void is created in the vertebral body by debulking the spinal tumor using the plasma radio-frequency-based wand before vertebral body augmentation with bone cement. $D-F$, Axial $(D$ and $E)$ and sagittal $(F)$ views by using MR imaging show excellent anterior placement of bone cement.

and preliminary outcomes by using this type of device to make a void in the affected vertebral body before inserting bone cement. With this procedure, they found it was possible to perform percutaneous cement augmentation in patients with advanced malignancy who would not normally be considered good candidates for undergoing conventional vertebroplasty or kyphoplasty. This technique was found to have the dual benefit of stabilizing the anterior column while minimizing the risk of anterior corpectomy by placing the injected cement in the anterior vertebral body, away from the compromised posterior wall (Fig 4). None of the patients treated in this series $(N=15)$ required subsequent open surgical treatment to palliate pain; all of them were managed conservatively with percutaneous cement augmentation in conjunction with radiation therapy.

A cavity may be created by using other methods such as balloon kyphoplasty. However, with balloon kyphoplasty, the cavity is created by tissue displacement, and tumor tissue may encroach on the central canal. These issues do not occur with radio-frequency-based plasma ablation because a cavity intended for bone cement placement is created when tumor tissue is removed.

\section{Recommendations for Treatment Selection}

Percutaneous cement augmentation by using either vertebroplasty or kyphoplasty is a safe procedure that is associated with successful palliation of pain and improved function in patients presenting with metastatic lesions of the spine. It is a beneficial adjuvant to radiation therapy and is an attractive alternative to surgery. Treating patients with vertebral compression fractures due to spinal malignancy is technically more demanding than treating patients with osteoporotic vertebral compression fractures, and many patients with malignant spinal lesions are not good candidates for conventional percutaneous cement augmentation procedures. Recent developments of the newer techniques described in the previous section appear to provide a safer means for treating these patients and fit well into the conventional oncologic treatment paradigm. An algorithm for percutaneous treatment of patients with spinal metastasis is presented in Fig 5.

Before any percutaneous procedure in patients with spinal malignancy, both CT and MR imaging should be performed. The MR imaging allows assessment of the degree of compression, epidural extension, paraspinal extension, presence of other lesions, and vascularity. The CT examination permits evaluation of vertebral body bony architecture and assessment of the posterior cortex and pedicles before augmentation, particularly in patients with cortical disruption and epidural extension. If there is the suggestion that the lesion is vascular, pretreatment embolization therapy may be considered. Neurologic monitoring should be considered in patients in whom the tumor extends into the pedicle.

Bony landmarks may not be clear under fluoroscopy, particularly when the tumor extends into the pedicles. The tip of 


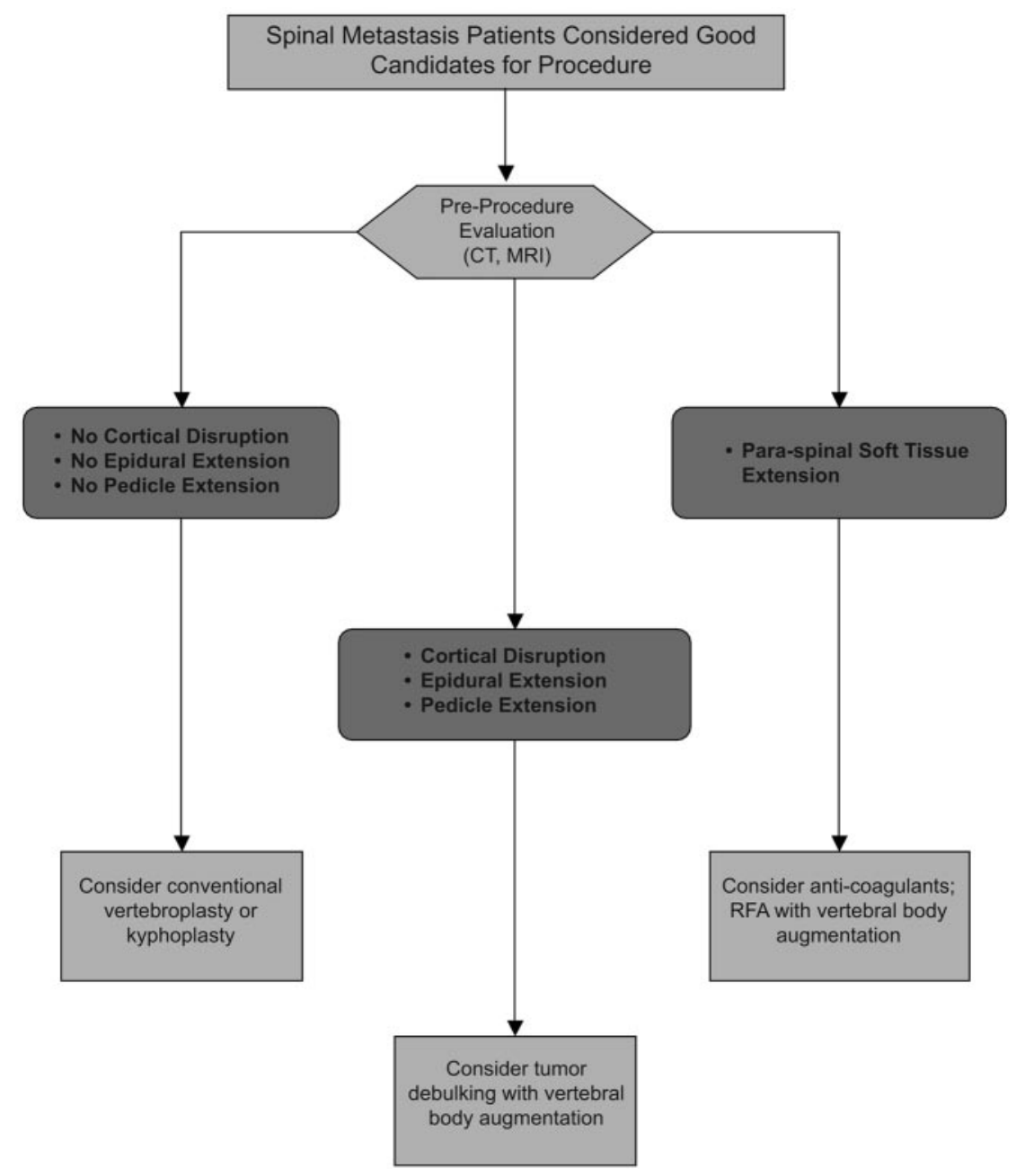

Fig 5. Candidates for percutaneous vertebral body augmentation who have spinal metastasis are best identified by evaluating the suggested treatment site by using CT and MR imaging (MRI). RFA indicates radio-frequency ablation.

the needle should remain lateral to the medial border of the pedicle. If the pedicle cannot be seen, a posterolateral approach can be used. Alternatively, the location of the pedicle can be extrapolated by using the pedicles of the vertebral bodies above and below the affected level or by using neurologic monitoring techniques. If necessary, the entire procedure can be performed under CT guidance. If the posterior cortex is not intact, myelography can be performed immediately before injecting the bone cement so that any movement of the tumor mass into the thecal sac or neural foramen can be avoided during augmentation. ${ }^{35,36}$ Vascular tumors, such as renal cell carcinomas, may be associated with frank blood flow through the cannula after removal of the stylet; the clinician must be prepared to lay bone cement along this track while withdrawing the needle to prevent excessive bleeding.

Placing bone cement into a vertebral body with an attenuated tumor mass is more difficult than placing cement into an osteoporotic vertebral body. Therefore, the bone cement pattern shown by CT may be spotty and discontinuous. ${ }^{10}$ Optimally, bone cement should be placed in the anterior aspect of the vertebral body so that maximal stability of the fractured vertebra can be achieved. Postprocedural examination by using CT to evaluate the position of the cement, changes in the position of the tumor mass, cement leakage, iatrogenic fracture, or unsuspected hematoma is advocated. ${ }^{10}$

\section{Opportunities for Future Improvements}

\section{Predicting Imminent Vertebral Body Compression Fracture}

Learning to recognize patients early in treatment who may be prone to sustaining a vertebral body compression fracture may facilitate clinical outcomes. Hiroshi et $\mathrm{al}^{37}$ found that impending vertebral body collapse could be predicted by using relatively simple guidelines. Evaluating the size and location of metastatic tumors by reviewing images of 100 thoracic and lumbar vertebrae with osteolytic lesions, they observed that compression fractures were most likely to occur under the following conditions: 1) 50\%-60\% involvement of the vertebral body with no destruction of other structures, 2) 35\%$40 \%$ involvement of vertebral body and $25 \%-30 \%$ with costovertebral joint destruction in the thoracic spine, and 3) $20 \%-25 \%$ involvement of the vertebral body with destruction of posterior elements in the thoracolumbar and lumbar spine. Routine evaluation by using these criteria when providing care for patients with impending vertebral body collapse may im- 
prove patient outcomes over simply waiting for the vertebral body to fracture and then considering treatment.

\section{Vertebral Body Augmentation with Hardware}

Vertebral body augmentation has been described previously as beneficial for treating traumatic burst fractures in which a short-segment pedicle screw fixation combined with vertebroplasty or kyphoplasty is used in lieu of traditional long-segment fusion. ${ }^{38}$ Acosta et $\mathrm{al}^{39}$ concluded that kyphoplasty supplementation may improve the long-term integrity of shortsegment pedicle screw constructs and allow improved rates of fusion and better clinical outcomes in patients with traumatic lumbar burst fractures. Bone cement-augmented screw fixations have been also described previously. ${ }^{40}$ Applications such as these could also potentially be developed for use in patients with bone malignancies, where tumor debulking is performed before placing hardware and augmentation.

\section{Conclusion}

Review of the current state of the art of percutaneous bone cement augmentation shows that this therapy provides a valuable addition to the current armamentarium for treating patients with vertebral body compression fractures associated with advanced spinal metastasis. Basic research of the biomechanical behavior of affected vertebral bodies indicates that imminent collapse can be predicted, suggesting new directions for understanding the pathophysiology and enabling the development of new therapies providing the clinician with the ability to specifically target the treatment site to apply the optimal treatment. Minimally invasive therapies are beneficial because complications and technical issues associated with open surgery are reduced and the number of patients ultimately receiving treatment earlier in the treatment algorithm will be increased.

\section{Acknowledgment}

I thank Debby Holmes-Higgin, MS, MPH, for technical assistance and stylistic editing of the manuscript.

\section{References}

1. Krishnaney AA, Steinmetz MP, Benzel EC. Biomechanics of metastatic spine cancer. Neurosurg Clin N Am 2004;15:375-80

2. Klimo P Jr, Schmidt MH. Surgical management of spinal metastases. Oncologist 2004;9:188-96

3. Ries LAG, Melbert D, Krapcho M, et al, eds. SEER Cancer Statistics Review, 1975-2005. National Cancer Institute. Bethesda, Md, Available at: http://seer. cancer.gov/csr/1975_2005/, based on November 2007 SEER data submission. Accessed August 7, 2007.

4. Klimo P, Schmidt MH. Surgical management of spine metastases. Oncologist 2004;9;188-96

5. Dimar JR, Voor MJ, Zhang YM, et al. A human cadaver model for determination of pathologic fracture threshold resulting from tumorous destruction of the vertebral body. Spine 1998;23:1209-14

6. Windhagen HJ, Hipp JA, Silva MJ, et al. Predicting failure of thoracic vertebrae with simulated and actual metastatic defects. Clin Orthop Relat Res 1997;313-19

7. Denis $F$. The three column spine and its significance in the classification of acute thoracolumbar spinal injuries. Spine 1983;8:817-31

8. Maranzano E, Latini P. Effectiveness of radiation therapy without surgery in metastatic spinal cord compression: final results from a prospective trial. Int J Radiat Oncol Biol Phys 1995;32:959-67

9. Wu AS, Fourney DR. Evolution of treatment for metastatic spine disease. $\mathrm{Neu}$ rosurg Clin N Am 2004;15:401-11

10. Jensen ME, Kallmes DE. Percutaneous vertebroplasty in the treatment of malignant spine disease. Cancer J 2002;8:194-206

11. Matsubayashi T, Koga H, Nishiyama Y. The reparative process of metastatic bone lesions after radiotherapy. Japan J Clin Oncol 1981;41:1039-47

12. Patel B, DeGroot $H$ 3rd. Evaluation of the risk of pathologic fractures secondary to metastatic bone disease. Orthopedics 2001;24:612-17
13. Regine WF, Tibbs PA, Young A, et al. Metastatic spinal cord compression: a randomized trial of direct decompression surgical resection plus radiotherapy vs. radiotherapy alone. International Journal of Radiation Oncology Biological Physics 2003;57:S125

14. Fourney DR, Gokaslan ZL. Anterior approaches for thoracolumbar metastatic spine tumors. Neurosurg Clin N Am 2004;15:443-51

15. Weinstein JN. Surgical approach to spine tumors. Orthopedics 1989;12:897-905

16. Harrington KD. The use of methylmethacrylate for vertebral-body replacement and anterior stabilization of pathological fracture-dislocations of the spine due to metastatic malignant disease. J Bone Joint Surg Am 1981;63:36-46

17. Cotten A, Dewatre F, Cortet B, et al. Percutaneous vertebroplasty for osteolytic metastases and myeloma: effects of the percentage of lesion filling and the leakage of methyl methacrylate at clinical follow-up. Radiology 1996;200:525-30

18. Weill A, Chiras J, Simon JM, et al. Spinal metastases: indications for and results of percutaneous injection of acrylic surgical cement. Radiology 1996;199:241-47

19. Deramond H, Depriester C, Galibert $P$, et al. Percutaneous vertebroplasty with polymethylmethacrylate: technique, indications, and results. Radiol Clin North Am 1998;36:533-46

20. Dudeney S, Lieberman IH, Reinhardt MK, et al. Kyphoplasty in the treatment of osteolytic vertebral compression fractures as a result of multiple myeloma. J Clin Oncol 2002;20:2382-87

21. Pflugmacher R, Kandziora F, Schroeder RJ, et al. Percutaneous balloon kyphoplasty in the treatment of pathological vertebral body fracture and deformity in multiple myeloma: a one-year follow-up. Acta Radiol 2006;47:369-76

22. Chiras J, Depriester C, Weill A, et al. Percutaneous vertebral surgery: techniques and indications [in French]. J Neuroradiol 1997;24:45-59

23. Mousavi P, Roth S, Finkelstein J, et al. Volumetric quantification of cement leakage following percutaneous vertebroplasty in metastatic and osteoporotic vertebrae. J Neurosurg 2003;99:56-59

24. Reidy D, Ahn H, Mousavi P, et al. A biomechanical analysis of intravertebra pressures during vertebroplasty of cadaveric spines with and without simulated metastases. Spine 2003;28:1534-39

25. Grönemeyer DH, Schirp S, Gevargez A. Image-guided radiofrequency ablation of spinal tumors: preliminary experience with an expandable array electrode. Cancer J 2002;8:33-39

26. Masala S, Roselli M, Massari F, et al. Radiofrequency heat ablation and vertebroplasty in the treatment of neoplastic vertebral body fractures. Anticancer Res 2004;24:3129-33

27. Schaefer O, Lohrmann C, Markmiller M, et al. Technical innovation: combined treatment of a spinal metastasis with radiofrequency heat ablation and vertebroplasty. AJR Am J Roentgenol 2003;180:1075-77

28. van der Linden E, Kroft LJ, Dijkstra PD. Treatment of vertebral tumor with posterior wall defect using image-guided radiofrequency ablation combined with vertebroplasty: preliminary results in 12 patients. J Vasc Interv Radiol 2007; 18:741-47

29. Goetz MP, Callstrom MR, Charboneau JW, et al. Percutaneous image-guided radiofrequency ablation of painful metastases involving bone: a multicenter study. J Clin Oncol 2004;22:300-06

30. Gevargez A, Grönemeyer DH. Image-guided radiofrequency ablation (RFA) of spinal tumors. Eur J Radiol 2008;65:246-52. Epub 2007 May 23

31. Buy X, Basile A, Bierry G, et al. Saline-infused bipolar radiofrequency ablation of high-risk spinal and paraspinal neoplasms. AJR Am J Roentgenol 2006; 186:S322-26

32. Tschirhart CE, Finkelstein JA, Whyne CM. Optimization of tumor volume reduction and cement augmentation in percutaneous vertebroplasty for prophylactic treatment of spinal metastases. J Spinal Disord Tech 2006;19:584-90

33. Ahn H, Mousavi P, Chin L, et al. The effect of pre-vertebroplasty tumor ablation using laser-induced thermotherapy on biomechanical stability and cement fill in the metastatic spine. Eur Spine J2007;16:1171-78. Epub 2007 Apr 20

34. Georgy BA, Wong W. Plasma-mediated radiofrequency ablation assisted percutaneous cement injection for treating advanced malignant vertebral compression fractures. AJNR Am J Neuroradiol 2007;28:700-05

35. Kanayama M, Ng JT, Cunningham BW, et al. Biomechanical analysis of anterior versus circumferential spinal reconstruction for various anatomic stages of tumor lesions. Spine 1999;24:445-50

36. Sarzier JS, Evans AJ. Intrathecal injection of contrast medium to prevent polymethylmethacrylate leakage during percutaneous vertebroplasty. AJNR Am J Neuroradiol 2003;24:1001-02

37. Hiroshi T, Kiyoshi K, Naoki T, et al. Risk factors and probability of vertebra body collapse in metastases of the thoracic and lumber spine. Spine 1997;22:239-45

38. Cho DY, Lee WY, Sheu PC. Treatment of thoracolumbar burst fractures with polymethyl methacrylate vertebroplasty and short-segment pedicle screw fixation. Neurosurgery 2003;53:1354-60, discussion 1360-61

39. Acosta FL Jr, Aryan HE, Taylor WR, et al. Kyphoplasty-augmented short-segment pedicle screw fixation of traumatic lumbar burst fractures: initial clinical experience and literature review. Neurosurg Focus 2005;18:e9

40. Jang JS, Lee SH, Rhee $\mathrm{CH}$, et al. Polymethylmethacrylate-augmented screw fixation for stabilization in metastatic spinal tumors: technical note. J Neurosurg 2002;96:131-34 